\title{
Educación virtual en tiempos de COVID-19: percepciones de estudiantes universitarios
}

\author{
William R. Avendaño', Henry O. Luna ${ }^{2}$ y Gerson Rueda ${ }^{3}$ \\ (1) Facultad de Ciencias Empresariales, Universidad Francisco de Paula Santander, Grupo de Investigación GICSH, \\ Cúcuta, Colombia. (Correo-e: williamavendano@ufps.edu.co) \\ (2) Facultad de Ciencias Empresariales, Universidad Francisco de Paula Santander, Grupo de Investigación ID \\ Investigación \& Desarrollo Regional, UFPS, Cúcuta, Colombia. (Correo-e: henryorlandolp@ufps.edu.co) \\ (3) Facultad de Ciencias Empresariales, Universidad Francisco de Paula Santander, Grupo de Investigación GIFOFI en \\ Formación Financiera, UFPS, Cúcuta, Colombia (Correo-e: gersonruedavera@ufps.edu.co)
}

Recibido Mar. 9, 2021; Aceptado May. 12, 2021; Versión final Jun. 16, 2021, Publicado Oct. 2021

\section{Resumen}

El objetivo de esta investigación es describir las percepciones de un grupo de estudiantes universitarios de nivel de pregrado en torno a la educación virtual en tiempos de COVID-19. El enfoque es cuantitativo, cuenta con un diseño no experimental-transversal, y es de tipo descriptivo. Se empleó como instrumento un cuestionario tipo Likert con 31 ítems, a una muestra de 608 estudiantes de la facultad de ciencias empresariales de una universidad pública de la ciudad de Cúcuta (Colombia). Los resultados muestran que la educación virtual en tiempos de COVID-19 para el escenario de investigación y la muestra de participantes ha sido adecuada y efectiva en las distintas dimensiones analizadas. Se concluye que la percepción en general de los estudiantes universitarios es favorable frente a la educación virtual, particularmente, respecto del papel que han tenido los docentes en cada uno de sus cursos y materias.

Palabras clave: COVID-19; percepciones; educación virtual; superior; pregrado

\section{Virtual education in times of COVID-19: perceptions of university students}

\begin{abstract}
The main objective of this research study is to describe the perceptions of a group of undergraduate students regarding virtual education in times of COVID-19. The study has a quantitative approach, a nonexperimental-cross-sectional design, and is descriptive. A Likert-type questionnaire with 31 items is used to sample 608 students enrolled at the faculty of business sciences of a public university in the city of Cúcuta (Colombia). The results show that virtual education in the times of COVID-19 for the research scenario and the sample of participants has been adequate and effective in the dimensions examined. It is concluded that the general perception of undergraduates about virtual education is favorable, in particular, with respect to the role that professors have played in each of their courses.
\end{abstract}

Keywords: COVID-19; perceptions; virtual education; higher; undergraduate 


\section{INTRODUCCIÓN}

La educación virtual es una tendencia actual tanto en las escuelas como en las instituciones de educación superior, y motivada, entre otros factores, por la necesidad o preferencia de las personas de aprender a través de entornos y modelos más flexibles (Martínez y Jiménez, 2020), las lógicas impuestas por la denominada sociedad del conocimiento, la información y el aprendizaje (Micheli y Armendáriz, 2011), la globalización y la urgencia de universalizar el derecho humano a la educación (Vélez, 2020). En Estados Unidos, por ejemplo, casi todos los estados ofrecen algún tipo de formación en línea, apoyada en tecnologías de la información y la comunicación, o combinada (Toppin y Toppin, 2016; Barbour, 2016; Molnar et al., 2019), en América Latina la denominada educación a distancia y virtual, crece de manera acelerada (Yong et al., 2017) y en Reino Unido hay escuelas totalmente virtuales con estructuras organizacionales plenamente definidas (Drew y Banerjee, 2019).

Por otro lado, las investigaciones que analizan las relaciones entre las tecnologías de la información y la comunicación, la educación y las instituciones educativas de distintos niveles, han aumentado de manera considerable desde el año 1995, principalmente, en los Estados Unidos, Reino Unido y China (Liu et al., 2017). El pronóstico de los investigadores y expertos es que la educación virtual y mediada por tecnologías, se incrementará en el futuro debido a sus utilidades y beneficios, impulsando transformaciones en distintos niveles, incluyendo los modelos pedagógicos y didácticos empleados por los docentes y exigidos para este tipo de escenarios de formación (Martín et al., 2017; Pando, 2018; Valkanov et al., 2016).

La educación virtual dentro de la literatura ha sido interpretada de distintas maneras. Micheli y Armendáriz (2011) describe esta categoría como aquel proceso de enseñanza y aprendizaje a través de Internet, ya sea de manera total o parcial. Dependiendo del uso pleno o parcial de Internet, la educación virtual puede ser sin presencia física del docente (e-learning) o combinada con tareas que incluyen interacción física y procesos apoyados en la Internet (b-learning). Vélez (2020) indica que la educación virtual o educación en línea hace referencia al conjunto de programas formativos donde la enseñanza y el aprendizaje tiene lugar en el ciberespacio, un lugar delimitado y configurado por tecnologías de la información y la comunicación, la conexión entre usuarios y metodologías pedagógicas-didácticas particulares.

Esta modalidad de educación delimitada y moldeada de forma permanente por las nuevas tecnologías, permiten diversificar la comunicación entre estudiantes y tutores, brinda una respuesta a las dificultades que surgen como resultado de los horarios y las distancias, aumenta la interacción entre los sujetos de formación, flexibiliza el proceso de enseñanza conforme a las necesidades e intereses de los participantes, y permite acceder a una gran cantidad de información de manera rápida y a bajo costo (Martínez y Jiménez, 2020). Igualmente, la educación virtual permite aprovechar un gran número de recursos tecnológicos como son los foros, chats o videoconferencias, potencia la autonomía de los sujetos de formación e impulsa la retroalimentación aprovechando el tiempo (Martínez y Garcés, 2020).

Sin embargo, pese a los beneficios y oportunidades que ofrece la educación virtual, también impone retos diversos a quienes hacen parte de este tipo de procesos. Tanto estudiantes como docentes deben desarrollar conocimientos y habilidades digitales (e-skills), responsabilidades, prácticas éticas y un alto nivel de criticidad para aprovechar el potencial aprendizaje en línea. Igualmente, los docentes deben capacitarse y emplear métodos alternativos para superar la pérdida de la interacción física entre los actores claves de estos procesos (Basantes et al., 2020; Huanca et al., 2020). Y desde una perspectiva institucional, se exige infraestructura, recursos y modelos organizacionales particulares.

En otras palabras, la incorporación de tecnologías de la información y la comunicación en el terreno educativo no garantiza de por sí el éxito de los procesos de enseñanza-aprendizaje en línea, pues hay todo un conjunto de requerimientos y condiciones que deben ser considerados y aplicados para alcanzar los objetivos de formación y la innovación educativa (Vélez, 2020). Incluso, se vienen definiendo modelos y métodos particulares para asegurar la calidad de la educación virtual desde comienzos de los años noventa del siglo veinte (Marciniak y Gairín, 2018).

Por lo anterior, la pandemia provocada por el Covid-19 (SARS-CoV2) significó una profunda crisis que se extendió de lo social y económico a otros terrenos como el educativo, develando las serias dificultades y limitaciones de las instituciones de formación, los docentes, los estudiantes y el sistema de educación en general. EI SARS-CoV2 fue informado por la Organización Mundial de la Salud, el 31 de diciembre de 2019 como un brote de neumonía en la provincia de Hubei en China (Perlman, 2020). En enero del 2020, el Covid-19 se convirtió en tema de interés mundial, lo que llevó a que las alarmas se prendieran por el potencial de contagio. El 11 de marzo de 2020, fue declarada la pandemia y se pasó de 66 mil casos en febrero a 17 millones de contagios al mes de julio de 2020 (Universidad Johns Hopkins, 2020). 
En el contexto colombiano, la pandemia obligó a que el 24 de marzo de 2020 se cerraran los aeropuertos y se decretó una cuarentena de 19 días. La pandemia llevó a profundas transformaciones en la forma de entender y percibir el mundo, así como en los modos de vivir. Además de los problemas sociales y económicos, los cambios y efectos se han extendido al terreno emocional por el confinamiento y el aislamiento, a los contenidos comunicativos y al campo de las interacciones de las personas (Van et al., 2020). Desde el origen de la pandemia, los estudiantes de instituciones de educación superior en Colombia, la gran mayoría acostumbrados al modelo de educación presencial, debieron afrontar procesos de enseñanza-aprendizaje en línea o medidas por tecnologías de la información y la comunicación, y claro está, tanto gobierno como instituciones educativas se vieron enfrentados a grandes retos y desafíos. Esta situación se encuentra extendida a nivel internacional, pues se estima que 1.300 millones de estudiantes en el mundo, se vieron afectados en sus procesos de educación como resultado de la pandemia (Bonilla, 2020).

En medio de estas nuevas dinámicas, las limitaciones y dificultades han evidenciado diferentes fenómenos en lo educativo: el escaso acceso a Internet y dispositivos en muchos estudiantes -manifestaciones de la inequidad y exclusión social- (Ahmady, et al., 2020; Huanca et al., 2020), las bajas competencias digitales de los docentes y estudiantes (Bonilla, 2020; Huanca et al., 2020), las dificultades para crear contenidos digitales y metodologías flexibles con cierto grado de innovación por parte de los docentes (Martínez y Garcés, 2020; Quintero, 2020), deficiencias en los medios y soportabilidad tecnológica de los actores de formación (Ahmady, et al., 2020; Huanca et al., 2020), las brechas entre instituciones educativas públicas y privadas en términos de recursos y capacidades organizacionales - operativas (Huanca et al., 2020). Otros estudios muestran las dificultades para adelantar los procesos de enseñanza-aprendizaje en determinadas disciplinas como la educación física (Baena, et al., 2021) o programas de educación superior como la medicina (Ehrlich, et al., 2020).

La pandemia del Covid-19 constituye, sin duda, un punto de inflexión, un hito histórico con irrupción en lo educativo. Dentro de este escenario, la educación virtual tiene un lugar significativo para la investigación educativa, pues se hace necesario explorar y analizar el fenómeno desde distintas perspectivas y aristas, particularmente, desde las percepciones de los actores educativos quienes pueden dar cuenta de los cambios y situación actual de la enseñanza, el aprendizaje, la didáctica, la pedagogía, entre otros elementos. En este estudio se analiza la educación virtual en tiempos del Covid-19 a partir de las percepciones de estudiantes universitarios de pregrado, en un intento por abonar al actual interés por indagar en las múltiples relaciones que se tejen entre educación, virtualidad y pandemia.

\section{METODOLOGÍA}

El estudio descrito en este artículo es de naturaleza cuantitativa de tipo no experimental-transversal y nivel descriptivo, enmarcado en el paradigma empírico analítico. Se empleó un método deductivo y un proceso de análisis de datos con estadística descriptiva. La población de la investigación estuvo integrada por 4.000 estudiantes de pregrado de la Facultad de Ciencias Empresariales de una universidad pública de la ciudad de Cúcuta, Norte de Santander (Colombia). A partir de la siguiente fórmula estadística para poblaciones conocidas o finitas, se calculó el tamaño de la muestra:

$$
\mathrm{n}=\frac{\mathrm{Zc}^{2} \times \mathrm{P} \times \mathrm{Q} \times}{\mathrm{E}^{2} \times(\mathrm{N}-1)+\mathrm{Zc^{2 }} \times \mathrm{P} \times \mathrm{Q}}
$$

Donde: $\mathrm{Zc}=95 \%$ o 1.96 es el nivel de certeza, bajo la curva normal; $P=0,5$ es la probabilidad de éxito; $Q=$ 0,5 es la probabilidad de fracaso; $E=5 \%-0,05$ es el nivel de error; y $N=4.000$ es la población

A partir de la fórmula estadística, se obtuvo una muestra de 351 estudiantes de pregrado. Los criterios de inclusión fueron: estudiantes matriculados en alguno de los programas de la Facultad, mayores de edad y haber visto clases virtuales o mediadas por TIC, por lo menos, durante tres meses. Los criterios de exclusión fueron: ser menores de edad, no tener la condición de matriculado en alguno de los programas de la Facultad en mención y tener menos de tres meses de experiencia en clases virtuales. Aunque la muestra fue calculada en 351 individuos, participaron en el desarrollo del instrumento un total 608 estudiantes, distribuidos de la siguiente forma: 237 estudiantes del programa Administración de Empresas (39\%), 201 estudiantes del programa Contaduría Pública (33\%) y 170 estudiantes de la Tecnología en Gestión Comercial y Financiera (28\%). 
El instrumento que se aplicó para la recolección de los datos correspondió a un cuestionario tipo Likert estructurado en 31 preguntas: 7 ítems relacionados con aspectos sociodemográficos y 24 distribuidas en dos variables. La primera de las variables se refiere a percepciones sobre la planeación y enseñanza, y la segunda a las percepciones en torno a los resultados de aprendizaje. La Tabla 1 muestra las variables y dimensiones en las que se dividieron los ítems del cuestionario:

Tabla 1: Estructura del cuestionario aplicado a estudiantes de pregrado de la Facultad de Ciencias Administrativas y Económicas de universidad pública (Cúcuta, Colombia)

\begin{tabular}{|c|c|c|}
\hline Variable & Dimensiones e indicadores & Preguntas \\
\hline \multirow{7}{*}{ Sociodemográfico } & Género & \multirow{7}{*}{$1-7$} \\
\hline & Edad & \\
\hline & Estrato socioeconómico & \\
\hline & Programa académico & \\
\hline & Semestre que cursa & \\
\hline & Residencia - Sector & \\
\hline & Condición especial & \\
\hline \multirow{23}{*}{$\begin{array}{l}\text { Percepción sobre la } \\
\text { planeación y la } \\
\text { enseñanza }\end{array}$} & Diseño del curso o la materia & \multirow{6}{*}{$8-12$} \\
\hline & - Organización y secuencias & \\
\hline & $-\quad$ Ritmos & \\
\hline & - $\quad$ Nivel de exigencia académica & \\
\hline & - $\quad$ Compromisos / tareas & \\
\hline & - $\quad$ Bibliografía & \\
\hline & Características de las clases & \multirow{6}{*}{$13-17$} \\
\hline & - Explicaciones del docente & \\
\hline & - $\quad$ Interés del estudiante & \\
\hline & - $\quad$ Motivación & \\
\hline & - $\quad$ Participación & \\
\hline & - $\quad$ Recursos y medios & \\
\hline & Calidad de la enseñanza & \multirow{7}{*}{$18-23$} \\
\hline & - $\quad$ Actitud docente & \\
\hline & - $\quad$ Empatía & \\
\hline & - $\quad$ Contenidos e información & \\
\hline & - $\quad$ Procedimientos organizados & \\
\hline & - $\quad$ Discurso docente & \\
\hline & - $\quad$ Manejo general de la clase & \\
\hline & Capacidades tecnológicas y sociales del docente & \multirow{4}{*}{$24-26$} \\
\hline & - $\quad$ Manejo de plataformas & \\
\hline & - $\quad$ Uso de redes & \\
\hline & - $\quad$ Recursos virtuales y digitales & \\
\hline \multirow{6}{*}{$\begin{array}{l}\text { Percepciones en } \\
\text { torno a los } \\
\text { resultados de } \\
\text { aprendizaje }\end{array}$} & Aprendizaje & \multirow{6}{*}{$27-31$} \\
\hline & - $\quad$ Autonomía del estudiante & \\
\hline & - $\quad$ Aprendizaje efectivo & \\
\hline & - $\quad$ Habilidades y competencias desarrolladas & \\
\hline & - $\quad$ Emociones & \\
\hline & - $\quad$ Satisfacción general & \\
\hline
\end{tabular}

Los ítems de las preguntas fueron planteados como afirmaciones y frente a cada uno de estos, los participantes de la muestra respondieron considerando cinco opciones de respuesta: muy de acuerdo, algo de acuerdo, ni de acuerdo ni en desacuerdo, algo en desacuerdo y muy en desacuerdo. Sin embargo, en la variable sociodemográfica se incluyeron opciones de respuesta diferentes. La validez del instrumento estuvo determinada por la técnica de juicio de expertos para el análisis de contenido. Participaron en el juicio de expertos, tres académicos con nivel de doctorado en el campo de la educación. Además, los evaluadores tenían más de 15 años de experiencia y contaban con distintas publicaciones científicas. Los criterios para la revisión y evaluación del instrumento fueron: claridad, coherencia, consistencia, neutralidad y suficiencia. La devolución de los expertos permitió efectuar ajustes al instrumento, se modificaron ítems conforme a las sugerencias dadas y se enviaron los instrumentos para una segunda revisión por parte de los expertos. Con la finalidad de evitar sesgos, se aplicó el instrumento a un número reducido de estudiantes universitarios 
que cursan iguales programas de formación, pero en otra sede de la misma universidad. A partir de este pilotaje, se logró establecer la claridad, neutralidad y suficiencia de los ítems que integran el instrumento diseñado y aplicado. Con estos datos se hizo un análisis de fiabilidad utilizado el alfa de Cronbach $(\alpha=1,00)$ mostrando que el instrumento es confiable. Posteriormente, se procedió a aplicar la prueba real a los estudiantes objeto de estudio.

Luego, el instrumento fue revisado por el Comité de Bioética de la Universidad para garantizar los aspectos éticos aplicables en las investigaciones, por ejemplo, la confidencialidad de los participantes. El cuestionario se subió a la plataforma Google Forms y se hizo la invitación a todos los estudiantes de la Facultad de Ciencias Empresariales entre los meses de octubre y noviembre de 2020, periodo en el que aumentaban de manera drástica el número de contagios por Coronavirus en Colombia -primer pico de la pandemia-. Pese a la situación, hubo buena recepción de los estudiantes al desarrollo del instrumento. Los datos recolectados fueron organizados, tabulados y analizados a través de métodos de estadística descriptiva apoyados en hojas de cálculo del programa Excel.

\section{RESULTADOS}

La Tabla 2 muestra las características y particularidades de la muestra de individuos que participo en la investigación. Se observa una prevalencia de mujeres (64\%) y un mayor porcentaje de estudiantes entre los 15 y 25 años (92\%). Respecto del estrato socioeconómico, el $50 \%$ de la muestra pertenece al estrato 2 y otro $38 \%$ al estrato 1 , es decir, la mayoría de la muestra tiene dificultades de acceso a recursos económicos. Los estudiantes que participaron en mayor porcentaje dentro de la muestra corresponden a los grupos de los semestres tres, cuatro, cinco y seis (66\%). El $95 \%$ de los estudiantes tiene su domicilio en zona urbana.

Tabla 2: Aspectos sociodemográficos de la muestra

\begin{tabular}{|c|c|c|c|}
\hline Dimensiones & Opciones & $N^{o}$ & $\%$ \\
\hline \multirow[t]{2}{*}{ Género } & Masculino & 220 & $36 \%$ \\
\hline & Femenino & 388 & $64 \%$ \\
\hline \multirow[t]{4}{*}{ Edad } & $15-20$ años & 317 & $52 \%$ \\
\hline & $21-25$ años & 241 & $40 \%$ \\
\hline & $26-30$ años & 28 & $5 \%$ \\
\hline & $31-45$ años & 22 & $4 \%$ \\
\hline \multirow[t]{4}{*}{ Nivel socioeconómico } & Estrato 1 & 230 & $38 \%$ \\
\hline & Estrato 2 & 301 & $50 \%$ \\
\hline & Estrato 3 & 74 & $12 \%$ \\
\hline & Estrato 4 & 3 & $1 \%$ \\
\hline \multirow[t]{3}{*}{ Programa } & Administración de Empresas & 237 & $39 \%$ \\
\hline & Contaduría Pública & 201 & $33 \%$ \\
\hline & Tecnología en Gestión Comercial y Financiera & 170 & $28 \%$ \\
\hline \multirow[t]{5}{*}{ Semestre } & 102 & 85 & $14 \%$ \\
\hline & 304 & 154 & $25 \%$ \\
\hline & 506 & 250 & $41 \%$ \\
\hline & 708 & 107 & $18 \%$ \\
\hline & $9 \circ 10$ & 12 & $2,0 \%$ \\
\hline \multirow[t]{2}{*}{ Residencia - Sector } & Zona urbana & 579 & $95 \%$ \\
\hline & Zona rural & 29 & $5 \%$ \\
\hline
\end{tabular}

La Tabla 3 muestra los resultados obtenidos en cada una de las dimensiones analizadas para la variable 'percepciones sobre la planeación y la enseñanza', claro está, todos los ítems contextualizados en el papel del docente en periodo de confinamiento y aislamiento como resultado de la pandemia en sus primeros meses en el 2020 (abril - septiembre).

Tabla 3: Resultados de la variable percepciones sobre la planeación y la enseñanza

\begin{tabular}{|c|c|c|c|c|}
\hline Dimensión & Pregunta & Opciones & No & $\%$ \\
\hline \multirow{8}{*}{$\begin{array}{l}\text { Diseño del } \\
\text { curso / materia }\end{array}$} & \multirow{5}{*}{$\begin{array}{l}\text { La organización y secuencia de los cursos o las } \\
\text { materias es adecuada y se ajusta a las } \\
\text { condiciones de la formación virtual. }\end{array}$} & Muy de acuerdo & 169 & $28 \%$ \\
\hline & & Algo de acuerdo & 278 & $46 \%$ \\
\hline & & Ni de acuerdo ni en desacuerdo & 93 & $15 \%$ \\
\hline & & Algo en desacuerdo & 51 & $8 \%$ \\
\hline & & Muy en desacuerdo & 17 & $3 \%$ \\
\hline & \multirow{3}{*}{$\begin{array}{l}\text { El ritmo de los cursos o las materias fue } \\
\text { adecuado y pertinente en beneficio de un } \\
\text { aprendizaje efectivo. }\end{array}$} & Muy de acuerdo & 154 & $25 \%$ \\
\hline & & Algo de acuerdo & 261 & $43 \%$ \\
\hline & & Ni de acuerdo ni en desacuerdo & 98 & $16 \%$ \\
\hline
\end{tabular}


Tabla 3: continuación

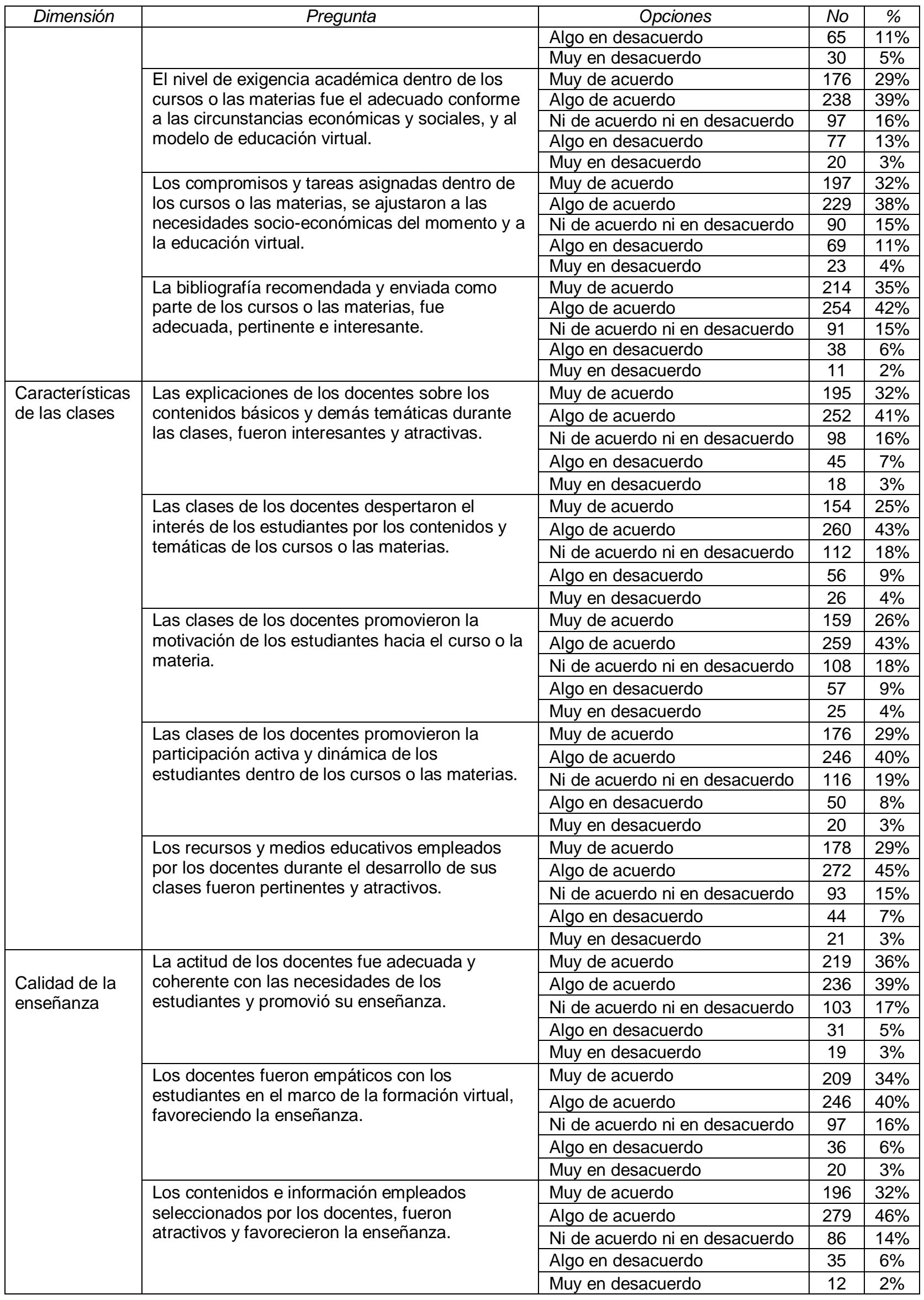


Tabla 3: continuación

\begin{tabular}{|c|c|c|c|c|}
\hline Dimensión & Pregunta & Opciones & No & $\%$ \\
\hline & \multirow{5}{*}{$\begin{array}{l}\text { Los procedimientos diseñados por los docentes } \\
\text { potencializaron la enseñanza de las habilidades } \\
\text { y competencias de los estudiantes. }\end{array}$} & Muy de acuerdo & 176 & $29 \%$ \\
\hline & & Algo de acuerdo & 273 & $45 \%$ \\
\hline & & Ni de acuerdo ni en desacuerdo & 115 & $19 \%$ \\
\hline & & Algo en desacuerdo & 28 & $5 \%$ \\
\hline & & Muy en desacuerdo & 16 & $3 \%$ \\
\hline & \multirow{5}{*}{$\begin{array}{l}\text { El discurso de los docentes (lenguaje expresivo) } \\
\text { durante sus clases, fue claro, preciso e } \\
\text { interesante. }\end{array}$} & Muy de acuerdo & 211 & $35 \%$ \\
\hline & & Algo de acuerdo & 264 & $43 \%$ \\
\hline & & Ni de acuerdo ni en desacuerdo & 81 & $13 \%$ \\
\hline & & Algo en desacuerdo & 38 & $6 \%$ \\
\hline & & Muy en desacuerdo & 14 & $2 \%$ \\
\hline & \multirow{5}{*}{$\begin{array}{l}\text { El manejo de las clases virtuales por parte de los } \\
\text { docentes, fue adecuado y satisfactorio. }\end{array}$} & Muy de acuerdo & 189 & $31 \%$ \\
\hline & & Algo de acuerdo & 270 & $44 \%$ \\
\hline & & Ni de acuerdo ni en desacuerdo & 95 & $16 \%$ \\
\hline & & Algo en desacuerdo & 41 & $7 \%$ \\
\hline & & Muy en desacuerdo & 13 & $2,1 \%$ \\
\hline \multirow{15}{*}{$\begin{array}{l}\text { Capacidades } \\
\text { tecnológicas y } \\
\text { sociales del } \\
\text { docente }\end{array}$} & \multirow{5}{*}{$\begin{array}{l}\text { Los docentes mostraron capacidades suficientes } \\
\text { para el manejo de las plataformas que mediaron } \\
\text { las clases. }\end{array}$} & Muy de acuerdo & 218 & $36 \%$ \\
\hline & & Algo de acuerdo & 259 & $43 \%$ \\
\hline & & Ni de acuerdo ni en desacuerdo & 88 & $14 \%$ \\
\hline & & Algo en desacuerdo & 30 & $5 \%$ \\
\hline & & Muy en desacuerdo & 13 & $2 \%$ \\
\hline & \multirow{5}{*}{$\begin{array}{l}\text { Los docentes mostraron capacidades suficientes } \\
\text { en el manejo de redes de comunicación para } \\
\text { favorecer el diálogo y los procesos formativos. }\end{array}$} & Muy de acuerdo & 219 & $36 \%$ \\
\hline & & Algo de acuerdo & 250 & $41 \%$ \\
\hline & & Ni de acuerdo ni en desacuerdo & 98 & $16 \%$ \\
\hline & & Algo en desacuerdo & 27 & $4 \%$ \\
\hline & & Muy en desacuerdo & 14 & $2 \%$ \\
\hline & \multirow{5}{*}{$\begin{array}{l}\text { Los docentes emplearon diferentes recursos } \\
\text { virtuales y digitales para apoyar el proceso de } \\
\text { formación con los estudiantes. }\end{array}$} & Muy de acuerdo & 215 & $35 \%$ \\
\hline & & Algo de acuerdo & 247 & $41 \%$ \\
\hline & & Ni de acuerdo ni en desacuerdo & 94 & $15 \%$ \\
\hline & & Algo en desacuerdo & 39 & $6 \%$ \\
\hline & & Muy en desacuerdo & 13 & $2 \%$ \\
\hline
\end{tabular}

Percepciones sobre el diseño del curso / materia: la mayoría de los estudiantes consideran que la organización y secuencia de los cursos se han ajustado a las condiciones de la educación virtual ( $28 \%$ muy de acuerdo y $46 \%$ algo de acuerdo). Igual criterio de percepción, mostraron los estudiantes frente al ritmo de los cursos o las materias (25\% muy de acuerdo y $43 \%$ algo de acuerdo). Igualmente, muy de acuerdo (29\%) y algo de acuerdo (39\%) son las opciones de mayor frecuencia entre los estudiantes que consideran que los cursos tienen el nivel de exigencia adecuada. La percepción de la mayoría de los participantes es que los compromisos académicos asignados se han ajustado a las necesidades socio-económicas del momento (muy de acuerdo $32 \%$ y algo de acuerdo $38 \%$ ), así como la bibliografía recomendada y que resulta pertinente (muy de acuerdo $35 \%$ y algo de acuerdo $42 \%$ ).

Percepciones sobre las características de las clases: los participantes coinciden en su mayoría que las explicaciones de los docentes han sido interesantes y atractivas (muy de acuerdo $32 \%$ y algo de acuerdo $41 \%$ ), así como que las clases han despertado el interés de los interlocutores (muy de acuerdo $25 \%$ y algo de acuerdo $43 \%$ ) y la motivación (muy de acuerdo $26 \%$ y algo de acuerdo $43 \%$ ). En el mismo sentido se encuentra la percepción de los estudiantes respecto de que los docentes han promovido la participación activa y dinámica (muy de acuerdo $29 \%$ y algo de acuerdo $40 \%$ ) y que han empleado recursos y medios educativos pertinentes (muy de acuerdo $29 \%$ y algo de acuerdo $45 \%$ ).

Percepciones sobre la calidad de enseñanza: la mayoría de los participantes consideran que la actitud de los docentes ha sido pertinente con las necesidades de los estudiantes (muy de acuerdo $36 \%$ y algo de acuerdo 39\%) y que estos han sido empáticos desde el escenario de la educación virtual (muy de acuerdo $34 \%$ y algo de acuerdo $40 \%$ ). En relación con los contenidos e información, la mayoría de los participantes consideran que estos han sido atractivos y han favorecido la enseñanza (muy de acuerdo $32 \%$ y algo de acuerdo $46 \%$ ), al igual que el discurso de los docentes (muy de acuerdo $35 \%$ y algo de acuerdo $43 \%$ ) y el manejo de las clases virtuales en general (muy de acuerdo $31 \%$ y algo de acuerdo $44 \%$ ). 
Percepción sobre las capacidades tecnológicas y sociales del docente. La mayoría de los participantes consideran que los docentes han mostrado capacidades suficientes en el manejo de las plataformas tecnológicas (muy de acuerdo $36 \%$ y algo de acuerdo 43\%), redes de comunicación apoyadas en las tecnologías (muy de acuerdo $36 \%$ y algo de acuerdo $41 \%$ ) y el empleo de recursos virtuales y digitales (muy de acuerdo $35 \%$ y algo de acuerdo $41 \%$ ). En la Tabla 4 se muestran los hallazgos de la percepción de los estudiantes en relación con los resultados de aprendizaje a través de la educación virtual en tiempos de Covid-19. La mayoría de los participantes considera que se ha promovido la autonomía a través de la educación virtual direccionada por los docentes (muy de acuerdo $34 \%$ y algo de acuerdo $43 \%$ ), así como el aprendizaje efectivo (muy de acuerdo $28 \%$ y algo de acuerdo $46 \%$ ) y el manejo-control de las emociones (muy de acuerdo $25 \%$ y algo de acuerdo $41 \%$ ). Igual percepción tiene los estudiantes sobre el desarrollo de las competencias, habilidades y destrezas que se promueven desde las materias o cursos (muy de acuerdo $26 \%$ y algo de acuerdo $45 \%$ ). En general, el $70 \%$ de los participantes se encuentran a satisfacción con el aprendizaje (muy de acuerdo $28 \%$ y algo de acuerdo $42 \%$ ).

Tabla 4: Resultados de la variable percepciones en torno a los resultados de aprendizaje

\begin{tabular}{|c|c|c|c|c|}
\hline Dimensión & Pregunta & Opciones & No & $\%$ \\
\hline \multirow[t]{15}{*}{ Aprendizaje } & \multirow{5}{*}{$\begin{array}{l}\text { Como resultado de la } \\
\text { enseñanza de los docentes en } \\
\text { el marco de la educación virtual, } \\
\text { se promovió la autonomía del } \\
\text { estudiante. }\end{array}$} & Muy de acuerdo & 206 & $34 \%$ \\
\hline & & Algo de acuerdo & 259 & $43 \%$ \\
\hline & & Ni de acuerdo ni en desacuerdo & 106 & $17 \%$ \\
\hline & & Algo en desacuerdo & 25 & $4 \%$ \\
\hline & & Muy en desacuerdo & 12 & $2 \%$ \\
\hline & \multirow{5}{*}{$\begin{array}{l}\text { Como resultado de la } \\
\text { enseñanza de los docentes en } \\
\text { el marco de la educación virtual, } \\
\text { se promovió el aprendizaje } \\
\text { efectivo de los estudiantes. }\end{array}$} & Muy de acuerdo & 169 & $28 \%$ \\
\hline & & Algo de acuerdo & 277 & $46 \%$ \\
\hline & & Ni de acuerdo ni en desacuerdo & 103 & $17 \%$ \\
\hline & & Algo en desacuerdo & 40 & $7 \%$ \\
\hline & & Muy en desacuerdo & 19 & $3 \%$ \\
\hline & \multirow{5}{*}{$\begin{array}{l}\text { Como resultado de la } \\
\text { enseñanza de los docentes en } \\
\text { el marco de la educación virtual, } \\
\text { los estudiantes desarrollaron las } \\
\text { habilidades y competencias } \\
\text { esperadas para el curso. }\end{array}$} & Muy de acuerdo & 161 & $26 \%$ \\
\hline & & Algo de acuerdo & 273 & $45 \%$ \\
\hline & & $\mathrm{Ni}$ de acuerdo ni en desacuerdo & 101 & $17 \%$ \\
\hline & & Algo en desacuerdo & 48 & $8 \%$ \\
\hline & & Muy en desacuerdo & 25 & $4 \%$ \\
\hline \multirow[t]{10}{*}{ Aprendizaje } & \multirow{5}{*}{$\begin{array}{l}\text { Como resultado de la } \\
\text { enseñanza de los docentes en } \\
\text { el marco de la educación virtual, } \\
\text { se promovió el manejo y control } \\
\text { de las emociones de los } \\
\text { estudiantes. }\end{array}$} & Muy de acuerdo & 151 & $25 \%$ \\
\hline & & Algo de acuerdo & 251 & $41 \%$ \\
\hline & & Ni de acuerdo ni en desacuerdo & 126 & $21 \%$ \\
\hline & & Algo en desacuerdo & 53 & $9 \%$ \\
\hline & & Muy en desacuerdo & 27 & $4 \%$ \\
\hline & \multirow{5}{*}{$\begin{array}{l}\text { Como resultado de la } \\
\text { enseñanza de los docentes en } \\
\text { el marco de la educación virtual, } \\
\text { me encuentro satisfecho con el } \\
\text { aprendizaje construido. }\end{array}$} & Muy de acuerdo & 172 & $28 \%$ \\
\hline & & Algo de acuerdo & 258 & $42 \%$ \\
\hline & & $\mathrm{Ni}$ de acuerdo ni en desacuerdo & 90 & $15 \%$ \\
\hline & & Algo en desacuerdo & 53 & $9 \%$ \\
\hline & & Muy en desacuerdo & 35 & $6 \%$ \\
\hline
\end{tabular}

\section{DISCUSIÓN}

A partid de los resultados obtenidos en la investigación, se evidencia que la educación virtual en tiempos de Covid-19 ha sido adecuada y efectiva en las distintas dimensiones analizadas para el escenario objeto de estudio. Un primer punto de discusión está dirigido a confirmar la incidencia positiva de la educación virtual en temas como el aprendizaje, la autonomía, el manejo de emociones, el desarrollo de competencias y habilidades en general. Los resultados de esta investigación no son coherentes con los hallazgos de otras investigaciones: Pérez et al. (2021) registra en una muestra de 548 universitarios en la Universidad de Extremadura en España que predomina la valoración negativa de la enseñanza a distancia por falta de adaptación de los docentes y modelos no centrados en el estudiante y Ramírez et al. (2020) con la participación de 183 estudiantes de la Universidad San Francisco Xavier de Chuquisaca en Bolivia, manifiesta que los sujetos participantes no tienen una buena percepción de la educación virtual por factores como el económico, la conectividad, el Internet y las metodologías de enseñanza.

Por otro lado, se pueden explicar los hallazgos obtenidos en la pronta adaptación que adelantaron los docentes universitarios frente a la urgencia de la educación virtual instalada en razón de la emergencia sanitaria. Otros estudios muestran que, pese a las debilidades y limitaciones de los docentes en materia de 
competencias digitales y tecnológicas, los profesores están haciendo especiales esfuerzos por dar respuesta a los estudiantes y estar a la altura de las exigencias del momento histórico (Velásquez, 2020). Esta adaptación no sólo se extiende a los docentes, pues algunas investigaciones recientes muestran que iguales procesos se han configurado en grupos de estudiantes universitarios (Gil et al., 2021).

En los resultados de esta investigación, no se brinda información sobre las decisiones y la inversión económica realizada por la dirección de la universidad para facilitar el tránsito a la educación virtual y la formación mediada por las TIC, pero los hallazgos permiten inferir que los docentes han dado cumplimiento a su misión formadora y que hay una satisfacción generalizada de los estudiantes. El Covid-19 ha impulsado el tránsito de la docencia y las universidades a nuevas realidades que exigen cambios y adaptaciones -pero sin transformaciones integrales- en cortos tiempos (García y Corell, 2020). En todo caso, se ha configurado la educación virtual como una tendencia y un fenómeno que ha llegado para quedarse (Cabero y Valencia, 2020).

Aunque los hallazgos de la investigación confirman que los docentes se han adaptado y modificado sus prácticas de enseñanza con apoyo de las tecnologías, dando continuidad a la formación, el diálogo, el desarrollo de actividades, la construcción de conocimiento y la reflexión sobre los problemas conceptuales y prácticos de cada una de las asignaturas, aún se requiere estudiar la adaptación de los estudiantes a estas nuevas lógicas de enseñanza a partir de sus capacidades reales limitados por el contexto social y económico que pueden afectar la disponibilidad de medios y recursos tecnológicos, la motivación, la conexión a Internet, el aprendizaje, entre otras dimensiones. Es cierto que los retos en el campo de la pedagogía son permanentes y que las prácticas de enseñanza deben actualizarse de manera constante, por lo que el Covid-19 y la pandemia deben ser asumidos como fenómenos que invitan a la flexibilidad permanente en la educación.

También se deberá señalar que hay un número de estudiantes universitarios insatisfechos con la virtualidad y la educación mediada por las tecnologías, y que no se pueden ignorar. Es probable que estas percepciones negativas sobre las distintas dimensiones analizadas, se deba a distintos factores como el aislamiento y las emociones que esto provoca, el quiebre a la tradicional formación presencial, a las dificultades pedagógicas por innovar en los espacios de enseñanza virtual, la falta del contacto humano, entre otros. Sin duda, hay retos presentes para los docentes universitarios en el marco de la educación virtual que se ha impuesto como resultado de la pandemia que exigen del reconocimiento de las necesidades estudiantiles, en especial, de aquellos que presentan dificultades para aprender y formarse en estos nuevos espacios.

\section{CONCLUSIONES}

La percepción en general de los estudiantes universitarios objeto de esta investigación es favorable frente a la educación virtual, particularmente, respecto del papel que han tenido los docentes en cada uno de sus cursos y materias. En consecuencia, se puede afirmar que los docentes universitarios están mostrando flexibilidad de adaptación frente a las exigencias del Covid-19 en el terreno educativo. La investigación adelantada muestra que los docentes están asumiendo un rol protagónico y dinámico en función de garantizar una enseñanza con pertinencia y coherencia respecto de las necesidades educativas de los estudiantes, así como las capacidades básicas requeridas para emplear los medios y recursos tecnológicos disponibles que facilitan la comunicación, el diálogo, la reflexión y el trabajo autónomo.

\section{REFERENCIAS}

Ahmady, S., Shahbazi, S., y Heidari, M., Transition to virtual learning during the coronavirus disease-2019 crisis in Iran: opportunity or challenge?, https://doi.org/10.1017/dmp.2020.142, Disaster Medicine and Public Health Preparedness, 14(3), 11-12 (2020)

Baena, S., López, J., y García, O., La intervención docente en educación física durante el periodo de cuarentena por COVID-19, https://doi.org/10.47197/retos.v0i39.80089, Retos, 39, 388-395 (2021)

Basantes, A.V., Cabezas, M., y Casillas, S., Competencias digitales en la formación de tutores virtuales en la Universidad Técnica del Norte, Ibarra-Ecuador, http://dx.doi.org/10.4067/S0718-50062020000500269, 13(5), 269-282 (2020)

Barbour, M.K., Virtual education: not yet ready for prime time? In the test-based education reforms: lessons from a failed agenda, $1^{\text {a }}$ Ed., 407-429, Information Age Publishing, Charlotte, NC, EEUU (2016)

Bonilla, J.A., Las dos caras de la educación en el COVID-19, http://dx.doi.org/10.33210/ca.v9i2.294, CienciAmérica, 9(2), 89-98 (2020) 
Cabero, J., y Valencia, R., And COVID-19 transformed the educational system: reflections and experiences to learn, https://doi.org/10.46661/ijeri.5246, IJERI: International Journal of Educational Research and Innovation, (15), 218-228 (2020)

Drew, H., y Banerjee, R., Supporting the education and well-being of children who are looked-after: what is the role of the virtual school? https://doi.org/10.1007/s10212-018-0374-0, European Journal of Psychology of Education, 34, 101-121 (2019)

Ehrlich, H., McKenney, M., y Elkbuli, A., We asked the experts: virtual learning in surgical education during the covid-19 pandemic-shaping the future of surgical education and training, https://doi.org/10.1007/s00268-020-05574-3, World J Surg, 44, 2053-2055 (2020)

García, F.J., y Corell, A., La Covid-19: ¿enzima de la transformación digital de la docencia o reflejo de una crisis metodológica y competencial en la educación superior?, Campus Virtuales, 9(2), 83-98 (2020)

Gil, R., León, A., y Calderón, D., Influence of Covid on the educational use of social media by students of teaching degrees, https://doi.org/10.14201/eks.23623, Education in the Knowledge Society, (22), 1-10 (2021)

Huanca, J.W., Supo, F., Sucari, R., y Supo, L.A., El problema social de la educación virtual universitaria en tiempos de pandemia, https://doi.org/10.22458/ie.v22iEspecial.3218, Innovaciones Educativas, 22(Especial), 115 - 128 (2020)

Liu, D., Bhagat, K.K., y otros tres autores, The potentials and trends of virtual reality in education, In Virtual, augmented, and mixed realities in education. Smart computing and intelligence, 1a Ed., 105-130, Springer, Singapore, Singapore (2017)

Marciniak, R., y Gairín, J., Dimensiones de evaluación de calidad de educación virtual: revisión de modelos referentes, https://doi.org/10.5944/ried.21.1.16182, Revista Iberoamericana de Educación a Distancia, 21(1), 1-15 (2018)

Martín, J, Mora, C.E., Añorbe, B., y González, A., Virtual technologies trends in education, https://doi.org/10.12973/eurasia.2017.00626a, J Math Sci Tech Ed., 13(2), 469-486 (2017)

Martínez, G.A., y Jiménez, N., Análisis del uso de las aulas virtuales en la Universidad de Cundinamarca, http://dx.doi.org/10.4067/S0718-50062020000400081, Formación Universitaria, 13(4), 81-92 (2020)

Martínez, J., y Garcés, J., Competencias digitales docentes y el reto de la educación virtual derivado de la covid-19, https://doi.org/10.17081/eduhum.22.39.4114, Educación y Humanismo, 22(39), 1-16 (2020)

Micheli, J., y Armendáriz, S., Estructuras de educación virtual en la organización universitaria. Un acercamiento a la sociedad del conocimiento, https://doi.org/10.4067/S0718-50062011000600005, Formación Universitaria, 4(6), 35-46 (2011)

Molnar, A., Miron, G., Elgeberi, N., y otros tres autores, Virtual schools in the U.S. 2019, 1a Ed., 7-10, National Education Policy Center, Boulder, EEUU (2019)

Pando, V.F., Teaching trends in virtual education: An interpretative approach, http://dx.doi.org/10.20511/pyr2018.v6n1.167, Propósitos y Representaciones, 6(1), 463-505 (2018)

Pérez, E., Vázquez, A., y Cambero, S., Educación a distancia en tiempos de COVID-19: Análisis desde la perspectiva de los estudiantes universitarios, http://dx.doi.org/10.5944/ried.24.1.27855, Revista Iberoamericana de Educación a Distancia RIED, 24(1), 331-350 (2021)

Perlman, S., Another decade, Another Coronavirus, https://doi.org/10.1056/NEJMe2001126, The New England Journal of Medicine, 382, 760-762 (2020)

Quintero, J. J., El efecto del COVID-19 en la economía y la educación: estrategias para la educación virtual de Colombia, https://doi.org/10.29394/Scientific.issn.2542-2987.2020.5.17.15.280-291, Revista Scientific, 5(17), 280-291 (2020)

Toppin, I.N., y Toppin, S.M., Virtual schools: the changing landscape of K-12 education in the U.S., https://doi.org/10.1007/s10639-015-9402-8, Education and Information Technologies, 21, 1571-1581 (2016)

Universidad Johns Hopkins, Mapa interactivo Covid-19 del Centro de Ciencia e Ingeniería de Sistemas (CSSE), tomado de https://coronavirus.jhu.edu/map.html (2020)

Valkanov, V., Stoyanov, S., y Valkanova, V., Virtual education space, https://doi.org/10.17265/1548-7709/2016.02.002, Journal of Communication and Computer, 13, 64-76 (2016)

Van, J., Baicker, K., y otros seis autores, Using social and behavioural science to support COVID-19 pandemic response, https://doi.org/10.1038/s41562-020-0884-z, Nature Human Behaviour, 4, 460-471 (2020)

Velásquez, R., La educación virtual en tiempos de Covid-19, https://doi.org/10.46734/revcientifica.v2i1.8, Revista Científica Internacional, 3(1), 19-25 (2020)

Vélez, R.M., Retos de las universidades latinoamericanas en la educación virtual, https://doi.org/10.35575/rvucn.n59a1, Revista Virtual Universidad Católica del Norte, (59), 1-3 (2020)

Yong, E., Nagles, N., Mejía, C., y Chaparro, C., Evolución de la educación superior a distancia: desafíos y oportunidades para su gestión, Revista Virtual Universidad Católica del Norte, (50), 81-105 (2017) 